archives

of thermodynamics

Vol. 38(2017), No. 2, 101-122

DOI: $10.1515 /$ aoter-2017-0012

\title{
Effect of rotation on a semiconducting medium with two-temperatures under L-S theory
}

\author{
MOHAMED I.A. OTHMAN ${ }^{a, b}$ \\ RAMADAN S. TANTAWI ${ }^{a}$ \\ EBTESAM E.M. ERAKI ${ }^{a *}$
}

a Department of Mathematics, Faculty of Science, P.O. Box 44519, Zagazig University, Zagazig, Egypt

$b$ Department of Mathematics, Faculty of Science, Taif University 888, Saudi Arabia

\begin{abstract}
The model of the equations of generalized thermoelasticity in a semi-conducting medium with two-temperature is established. The entire elastic medium is rotated with a uniform angular velocity. The formulation is applied under Lord-Schulman theory with one relaxation time. The normal mode analysis is used to obtain the expressions for the considered variables. Also some particular cases are discussed in the context of the problem. Numerical results for the considered variables are obtained and illustrated graphically. Comparisons are also made with the results predicted in the absence and presence of rotation as well as two-temperature parameter.
\end{abstract}

Keywords: Normal mode analysis; Lord-Shulman theory; Rotation; Conductive temperature; Semiconductors

\section{Nomenclature}

$$
\begin{array}{lll}
a^{*} & - & \text { two temperature parameter } \\
c_{e} & - & \text { specific heat at constant strain } \\
D_{E} & - & \text { carrier diffusion coefficient }
\end{array}
$$

${ }^{*}$ Corresponding Author. Email ebtesam.eraki@yaho.com 


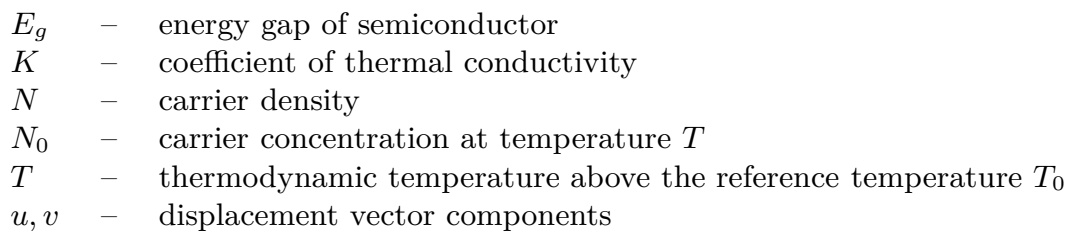

\section{Greek symbols}

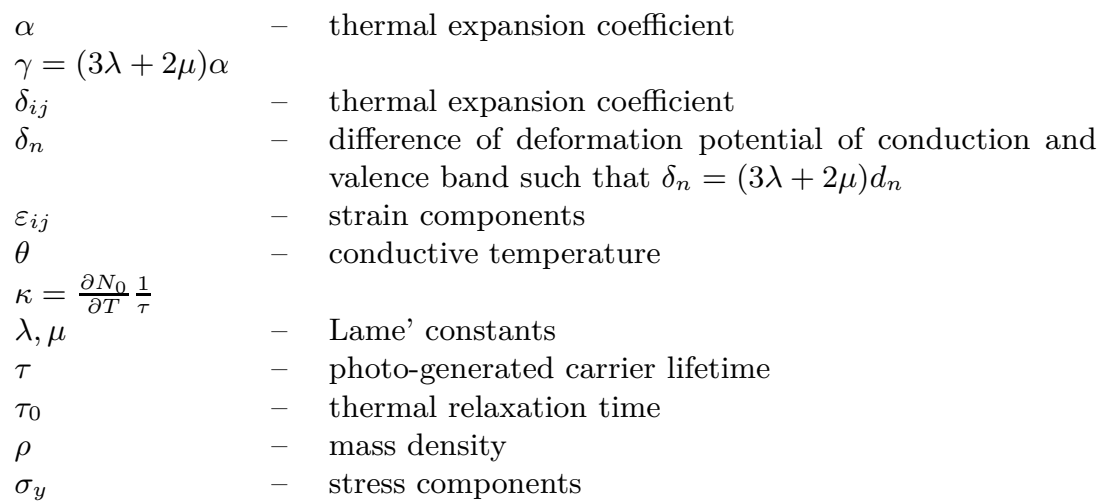

\section{Introduction}

Thermoelasticity theories, which admit a finite speed for thermal signals, have received a lot of attention for the past four decades. In contrast to the coupled thermo-elasticity theory based on a parabolic heat equation [1], which predicts an infinite speed of the propagation of heat, these theories involve a hyperbolic heat equation and are referred to as generalized thermoelasticity theories. The theory of thermoelasticity with one relaxation time proposed by Lord and Shulman [2] arose as a result of the modification of equation of heat conduction in [1], originally proposed by Maxwell [3] in the context of theory of gases, and later by Cattaneo [4] and Vernotte [5] in the context of heat conduction in rigid bodies. Resulting from that theory heat equation of the wave type ensures the finite speed of wave propagation of heat and the displacement distributions. This theory was extended by Dhaliwal and Sherief [6] to include the anisotropic case. Othman and Said [7] used the normal mode analysis to study the effect of rotation on the two-dimensional problem of a fibre-reinforced thermoelastic with one relaxation time.

The first photothermal method was discovered by Gordon et al. [8] when they found the intracavity sample where a laser-based apparatus gave 
rise to photothermal blooming, namely the photothermal lens. Sometime later, Kreuzer [9] showed that photoacoustic spectroscopy could be used for sensitive analysis when laser light sources were used. The photo-thermal generation during a photothermal process was studied by many authors. For semiconductor materials, the mechanism of this process includes two parts: two parts: part one: the propagation of a thermal wave causing elastic vibration in the medium; this is the thermoelastic (TE) mechanism of photothermal generation (Todorovic et al. [10]). Part two: the photoexcite free carriers produce directly a periodic elastic deformation that is, the electronic deformation (ED) in the sample (Todorovic et al. [10]). A general theoretical analysis of the (TE) and (ED) effects in a semi-conductor medium during a photo-thermal process consists in modeling the complex systems by simultaneous analysis of the coupled plasma, thermal, and elastic wave equations (Song et al. [11]). System of partially coupled plasma, thermal and elastic wave equations and conditions for neglecting the coupling between them is analyzed (Todorovic [12]). The treatment considers a semiconductor elastic medium for isotropic and homogeneous, thermal and elastic properties. Song et al. [13] used the coupled generalized thermoelastic with thermal relaxation time and plasma theories to study the reflection problem at the surface of a semi-infinite semiconducting medium during a photothermal process.

Some research in the past investigated different problems of rotating media. In a paper by Schoenberg and Censor [14], the propagation of plane harmonic waves in a rotating elastic medium without a thermal field has been studied. It was shown there that the rotation causes the elastic medium to be depressive and anisotropic. Many authors [15-25] studied the effect of rotation on elastic waves. These problems are based on the more realistic elastic model since earth, the moon and other planets have angular velocity.

Thermoelasticity with two temperatures is one of the nonclassical theories of thermoelasticity of elastic solids. The thermal dependence is the main difference of this theory with respect to the classical one. Chen and Gurtin [26], Chen et al. [27,28] have formulated a theory of heat conduction in deformable bodies, which depend on two distinct temperatures, the conductive temperature and thermodynamic temperature. For time independent situations, the difference between these two temperatures is proportional to the heat supply. For time dependent problems and with respect to wave propagation problem in particular, the two-temperatures 
are in general different, regardless of the presence of heat supply. The two temperatures, $T$, and $\theta$, and the strain are found to have representations in the form of a traveling wave plus a response, which occurs instantaneously throughout the body [29]. Warren and Chen [30] investigated the wave propagation in the two-temperature theory of thermoelasticity. Recently, Youssef [31,32], Abbas and Youssef [33] and Bijarnia and Singh [34] studied different problems under two temperature generalized thermoelastic theory.

This paper investigates the effect of two-temperature parameter and rotation in a semiconducting medium into the context of the two-temperature generalized thermoelasticity theory with one relaxation time.

\section{Formulation of the problem and basic equations}

Generally, theoretical analyses of the transport process in a semiconductor involve in the consideration of coupled plasma waves, thermal waves and elastic waves simultaneously. For a medium with isotropic and homogeneous properties, when the body forces are neglected, the governing equations are; Fig. 1:

1. Strain-displacement relations:

$$
\varepsilon_{i j}=\frac{1}{2}\left(u_{i, j}+u_{j, i}\right), \quad i, j=1,2,
$$

where the components of the displacement vector are $\mathbf{u} \equiv(u, v, 0)$.

2. Constitutive relations:

$$
\sigma_{i j}=2 \mu \varepsilon_{i j}+\left[\lambda e-\gamma T-\delta_{n} N\right] \delta_{i j} .
$$

3. Heat conduction equation (hyperbolic equation [35]):

$$
K \nabla^{2} \theta+\frac{E_{g}}{\tau} N-\gamma T_{0}\left(1+\tau_{0} \frac{\partial}{\partial t}\right) \dot{e}=\rho c_{e}\left(1+\tau_{0} \frac{\partial}{\partial t}\right) \dot{T},
$$

such that

$$
T=\theta-a^{*} \nabla^{2} \theta
$$

4. Equation of motion:

Since the medium is rotating uniformly with an angular velocity $\boldsymbol{\Omega}=$ $\Omega n \equiv(0,0, \Omega)$ where $n$ is a unit vector representing the direction of 
the axis of the rotation, the equation of motion in the rotating frame of reference has two additional terms (Schoenberg and Censor [14]): centripetal acceleration $\boldsymbol{\Omega} \times(\Omega \times \mathbf{u})$ due to time-varying motion only and Corioli's acceleration $2 \Omega \times \dot{\mathbf{u}}$, then the equation of motion in a rotating frame of reference is

$\mu \nabla^{2} u_{i}+(\lambda+\mu) \nabla e-\gamma \nabla T-\delta_{n} \nabla N=\rho\left[\ddot{u}_{i}+[\Omega \times \Omega \times \mathbf{u}]_{i}+2(\boldsymbol{\Omega} \times \dot{\mathbf{u}})_{i}\right]$.

5. Coupled plasma transport equation (parabolic equation [35]):

$$
D_{E} \nabla^{2} N-\frac{N}{\tau}+\kappa T=\frac{\partial N}{\partial t},
$$

where $N$ is the carrier density, $T$ is the thermodynamic temperature above the reference temperature $T_{0}, \sigma_{i j}$ are the stress components, $\varepsilon_{i j}$ are the strain components, $\lambda, \mu$ are Lame' constants, $\gamma=(3 \lambda+$ $2 \mu) \alpha_{t}, \alpha_{t}$ is the thermal expansion coefficient, $\delta_{n}$ is the difference of deformation potential of conduction and valence band such that $\delta_{n}=(3 \lambda+2 \mu) d_{n}, \delta_{i j}$ is the Kronecker delta, $K$ is the coefficient of thermal conductivity, $\theta$ is the conductive temperature, $a^{*}$ is the two temperature parameter, $\rho$ is the mass density, $c_{e}$ is the specific heat at constant strain, $E_{g}$ is the energy gap of semiconductor, $D_{E}$ is the carrier diffusion coefficient, $\tau$ is the photo-generated carrier lifetime, $\tau_{0}$ is the thermal relaxation time and $\kappa=\frac{\partial N_{0}}{\partial T} \frac{1}{\tau}, \quad N_{0}$ is the carrier concentration at temperature $T$.

The governing equations can be put into a more convenient form by using the following non-dimensional variables:

$$
\begin{gathered}
\left(x^{\prime}, y^{\prime}, u^{\prime}, v^{\prime}\right)=\frac{1}{C_{T} t^{*}}(x, y, u, v), \quad\left(t^{\prime}, \tau_{0}^{\prime}\right)=\frac{1}{t^{*}}\left(t, \tau_{0}\right), \\
\left\{T^{\prime}, \theta^{\prime}\right\}=\frac{\gamma}{(\lambda+2 \mu)}\{T, \theta\}, \quad N^{\prime}=\frac{\delta_{n}}{(\lambda+2 \mu)} N, \\
\sigma_{i j}^{\prime}=\frac{1}{\mu} \sigma_{i j}, \Omega^{\prime}=t^{*} \Omega, \quad C_{T}^{2}=\frac{(\lambda+2 \mu)}{\rho}, \quad t^{*}=\frac{K}{\rho c_{e} C_{T}^{2}} .
\end{gathered}
$$

Introducing the displacement potentials $\Phi(x, y, t)$ and $\Psi(x, y, t)$, which related to displacement components by the relations

$$
u=\Phi_{, x}+\Psi_{, y} \quad v=\Phi_{, y}-\Psi_{, x} .
$$




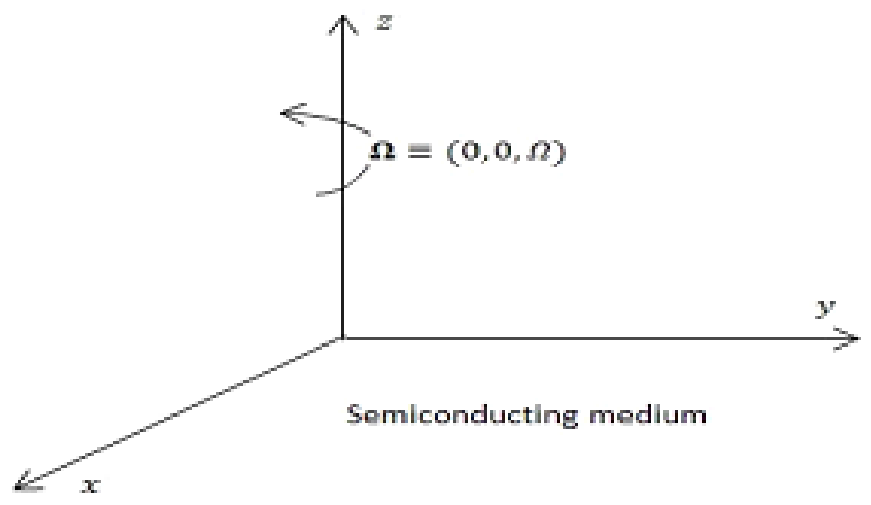

Figure 1: Schematic diagram of the problem.

Using Eqs. (2) and (8), in Eqs. (3)-(6), we obtain (the dashed above quantities have been removed for convenience):

$$
\begin{gathered}
{\left[\nabla^{2}+\Omega^{2}-\frac{\partial^{2}}{\partial t^{2}}\right] \Phi-2 \Omega \frac{\partial \Psi}{\partial t}-\left(1-a_{0} \nabla^{2}\right) \theta-N=0} \\
2 \Omega \beta^{2} \frac{\partial \Phi}{\partial t}+\left[\nabla^{2}+\beta^{2} \Omega^{2}-\beta^{2} \frac{\partial^{2}}{\partial t^{2}}\right] \Psi=0 \\
\left\{\left[1+a_{0}\left(1+\tau_{0} \frac{\partial}{\partial t}\right) \frac{\partial}{\partial t}\right] \nabla^{2}-\left(1+\tau_{0} \frac{\partial}{\partial t}\right) \frac{\partial}{\partial t}\right\} \theta-\varepsilon_{1}\left(1+\tau_{0} \frac{\partial}{\partial t}\right) \nabla^{2} \dot{\Phi}+\varepsilon_{2} N=0 \\
{\left[\nabla^{2}-\frac{K t^{*}}{\rho c_{e} \tau D_{E}}-\frac{K}{\rho c_{e} D_{E}} \frac{\partial}{\partial t}\right] \mathrm{N}+\varepsilon_{3}\left(1-a_{0} \nabla^{2}\right) \theta=0} \\
\mathrm{~T}=\left(1-a_{0} \nabla^{2}\right) \theta .
\end{gathered}
$$

For the stress-tensor components, we have the following expressions:

$$
\begin{gathered}
\sigma_{x x}=\beta^{2} u_{, x}+\left(\beta^{2}-2\right) v_{, y}-\beta^{2} T-\beta^{2} N, \\
\sigma_{y y}=\left(\beta^{2}-2\right) u_{, x}+\beta^{2} v_{, y}-\beta^{2} T-\beta^{2} N, \\
\sigma_{z z}=\left(\beta^{2}-2\right) \nabla^{2} \Phi-\beta^{2} T-\beta^{2} N, \\
\sigma_{x y}=u_{, y}+v_{, x}, \quad \sigma_{x z}=\sigma_{y z}=0,
\end{gathered}
$$

where:

$\varepsilon_{1}=\frac{\gamma^{2} T_{0} t^{*}}{K \rho}, \varepsilon_{2}=\frac{\alpha_{t} E_{g} t^{*}}{\rho c_{e} \tau d_{n}}, \varepsilon_{3}=\frac{K \kappa d_{n} t^{*}}{\rho c_{e} \alpha_{t} D_{E}}, \beta^{2}=\frac{(\lambda+2 \mu)}{\mu}$, and $a_{0}=\frac{a^{*}}{C_{T}^{2} t^{* 2}}$. 


\section{Normal mode analysis}

The solution of considered physical quantities can be decomposed in terms of normal mode as follows:

$\left[\Phi, \Psi, T, \theta, \mathrm{N}, \sigma_{i j}\right](x, y, t)=\left[\Phi^{*}, \Psi^{*}, T^{*}, \theta^{*}, N^{*}, \sigma_{i j}^{*}\right](y) \exp (\omega t+i a x)$,

where $a$ is the wave number in the $x$-direction, $i=\sqrt{-1}, \omega$ is a complex constant, $\Phi^{*}, \Psi^{*}, \mathrm{~T}^{*}, \theta^{*}, N^{*}$, and $\sigma_{i j}^{*}$ are the amplitudes of the field quantities $\Phi, \Psi, \mathrm{T}, \theta, N$, and $\sigma_{i j}$.

Using Eq. (18), Eqs.(9)-(12) become respectively:

$$
\begin{gathered}
\left(D^{2}-b_{1}\right) \Phi^{*}-b_{2} \Psi^{*}+\left(a_{0} D^{2}-b_{3}\right) \theta^{*}-N^{*}=0, \\
b_{4} \Phi^{*}+\left(\mathrm{D}^{2}-b_{5}\right) \Psi^{*}=0, \\
-b_{6}\left(D^{2}-a^{2}\right) \Phi^{*}+\left(b_{7} D^{2}-b_{8}\right) \theta^{*}+\varepsilon_{2} N^{*}=0, \\
\left(b_{9} D^{2}-b_{10}\right) \theta^{*}-\left(D^{2}-b_{11}\right) N^{*}=0,
\end{gathered}
$$

where:

$$
\begin{aligned}
& b_{1}=a^{2}+\omega^{2}-\Omega^{2}, \quad b_{2}=2 \omega \Omega, \quad b_{3}=\left(1+a_{0} a^{2}\right), \quad b_{4}=b_{2} \beta^{2}, \\
& b_{5}=a^{2}+\beta^{2}\left(\omega^{2}-\Omega^{2}\right), \quad b_{6}=\varepsilon_{1} \omega\left(1+\tau_{0} \omega\right), \quad b_{7}=1+a_{0} \omega\left(1+\tau_{0} \omega\right), \\
& b_{8}=a^{2} b_{7}+\omega\left(1+\tau_{0} \omega\right), \quad b_{9}=a_{0} \varepsilon_{3}, \quad b_{10}=\varepsilon_{3}\left(1+a_{0} a^{2}\right), \quad b_{11}=a^{2}+\alpha, \\
& \alpha=\frac{K t^{*}}{\rho c_{e} \tau D_{E}}+\frac{K \omega}{\rho c_{e} D_{E}}, \quad D=\frac{d}{d y} .
\end{aligned}
$$

Eliminating $\Phi^{*}(y), \Psi^{*}(y), \theta^{*}(y)$, and $N^{*}(y)$ between Eqs. (19)-(22), the following eighth order ordinary differential equation satisfied by $\Phi^{*}(y), \Psi^{*}(y)$, $\theta^{*}(y)$, and $N^{*}(y)$ can be obtained:

$$
\left(D^{8}-A D^{6}+B D^{4}-C D^{2}+E\right)\left[\Phi^{*}(y), \Psi^{*}(y), \theta^{*}(y), N^{*}(y)\right]=0,
$$

where:

$$
\begin{aligned}
& \begin{aligned}
A= & \frac{1}{\left(a_{0} b_{6}+b_{7}\right)}\left[\left(b_{1}+b_{5}+b_{11}\right) b_{7}+b_{8}-\left(\varepsilon_{2}-b_{6}\right) b_{9}+a_{0} b_{6}\left(a^{2}+b_{11}\right)\right. \\
\quad & \left.+b_{6}\left(a_{0} b_{5}+b_{3}\right)\right],
\end{aligned} \\
& B=\frac{1}{\left(a_{0} b_{6}+b_{7}\right)}\left[b_{3} b_{5} b_{6}+b_{8} b_{11}-\varepsilon_{2} b_{10}+\left(b_{1}+b_{5}\right)\left(b_{7} b_{11}+b_{8}-\varepsilon_{2} b_{9}\right)+\right. \\
& \left.\left(b_{1} b_{5}+b_{2} b_{4}\right) b_{7}+a^{2} b_{6}\left(a_{0} b_{11}+b_{9}\right)+b_{6}\left(a_{0} b_{5}+b_{3}\right)\left(a^{2}+b_{11}\right)+b_{6}\left(b_{5} b_{9}+b_{10}\right)\right], \\
& C=\frac{1}{\left(a_{0} b_{6}+b_{7}\right)}\left[\left(b_{1}+b_{5}\right)\left(b_{8} b_{11}-\varepsilon_{2} b_{10}\right)+\left(b_{1} b_{5}+b_{2} b_{4}\right)\left(b_{7} b_{11}+b_{8}-\varepsilon_{2} b_{9}\right)\right.
\end{aligned}
$$


$E=\frac{1}{\left(a_{0} b_{6}+b_{7}\right)}\left[\left(b_{1} b_{5}+b_{2} b_{4}\right)\left(b_{8} b_{11}-\varepsilon_{2} b_{10}\right)+a^{2} b_{5} b_{6}\left(b_{3} b_{11}+b_{10}\right)\right]$.

Equation (23) can be factored as

$$
\left(\mathrm{D}^{2}-k_{1}^{2}\right)\left(\mathrm{D}^{2}-k_{2}^{2}\right)\left(\mathrm{D}^{2}-k_{3}^{2}\right)\left(\mathrm{D}^{2}-k_{4}^{2}\right)\left\{\Phi^{*}(y), \Psi^{*}(y), \theta^{*}(y), \mathrm{N}^{*}(y)\right\}=0,
$$

where $k_{j}^{2}(j=1,2,3,4)$ are the roots of the characteristic equation given by Eq. (24). The limit of Eq. (24), solution as $y \rightarrow \infty$, reads:

$$
\begin{gathered}
\Phi^{*}(y)=\sum_{n=1}^{4} M_{n} e^{-k_{n} y}, \\
\Psi^{*}(y)=\sum_{n=1}^{4} H_{1 n} M_{n} e^{-k_{n} y}, \\
\theta^{*}(y)=\sum_{n=1}^{4} H_{2 n} M_{n} e^{-k_{n} y}, \\
N^{*}(y)=\sum_{n=1}^{4} H_{3 n} M_{n} e^{-k_{n} y},
\end{gathered}
$$

here $M_{n}(n=1,2,3,4)$ are some coefficients and $H_{1 \mathrm{n}}=\frac{b_{4}}{\left(b_{5}-k_{n}^{2}\right)}$,

$H_{2 \mathrm{n}}=\frac{b_{6}\left(k_{n}^{2}-a^{2}\right)\left(k_{n}^{2}-b_{11}\right)}{\left[\left(k_{n}^{2}-b_{11}\right)\left(b_{7} k_{n}^{2}-b_{8}\right)+\varepsilon_{2}\left(b_{9} k_{n}^{2}-b_{10}\right)\right]}, H_{3 \mathrm{n}}=\frac{\left(b_{9} k_{n}^{2}-b_{10}\right) H_{2 n}}{\left(k_{n}^{2}-b_{11}\right)}$.

Using Eqs. (8), (13), (18), and (25)-(28), the displacement components and the thermo-dynamic temperature can be obtained in the following form:

$$
\begin{gathered}
u^{*}(y)=\sum_{n=1}^{4} v_{1 n} M_{n} e^{-k_{n} y}, \\
v^{*}(y)=-\sum_{n=1}^{4} v_{2 n} M_{n} e^{-k_{n} y}, \\
T^{*}(y)=\sum_{n=1}^{4} v_{3 n} M_{n} e^{-k_{n} y} .
\end{gathered}
$$

Using Eqs. (14)-(18) and (25)-(31), we obtain

$$
\sigma_{x x}^{*}=\sum_{n=1}^{4} H_{4 n} M_{n} e^{-k_{n} y},
$$




$$
\begin{gathered}
\sigma_{y y}^{*}=\sum_{n=1}^{4} H_{5 n} M_{n} e^{-k_{n} y}, \\
\sigma_{z z}^{*}=\sum_{n=1}^{4} H_{6 n} M_{n} e^{-k_{n} y}, \\
\sigma_{x y}^{*}=-\sum_{n=1}^{4} H_{7 n} M_{n} e^{-k_{n} y}, \sigma_{x z}^{*}=\sigma_{y z}^{*}=0,
\end{gathered}
$$

where: $v_{1 n}=i a-k_{n} H_{1 n}, \quad v_{2 n}=k_{n}+i a H_{1 n}, \quad v_{3 n}=\left[1-a_{0}\left(k_{n}^{2}-a^{2}\right)\right] H_{2 n}$, $H_{4 n}=\left(\beta^{2}-2\right) k_{n} v_{2 n}+\beta^{2}\left(i a v_{1 n}-v_{3 n}-H_{3 n}\right), H_{5 n}=i a\left(\beta^{2}-2\right) v_{1 n}+$ $\beta^{2}\left(k_{n} v_{2 n}-v_{3 n}-H_{3 n}\right), \quad H_{6 n}=\left(\beta^{2}-2\right)\left(k_{n}^{2}-a^{2}\right)-\beta^{2}\left(v_{3 n}+H_{3 n}\right)$, $H_{7 n}=k_{n} v_{1 n}+i \_a v_{2 n}$.

\section{Boundary conditions}

The coefficients $M_{n}(n=1,2,3,4)$ have to be chosen such that the boundary conditions on the surface $y=0$, take the form

$$
\begin{gathered}
\sigma_{x x}=-P_{1}^{*} \exp (\omega t+i a x), \quad \sigma_{x y}=0, \\
T=P_{2}^{*} \exp (\omega t+i a x), \\
D_{E} \frac{d N}{d y}=s N,
\end{gathered}
$$

where $P_{1}^{*}, P_{2}^{*}$, and $s$ are constants.

Applying the boundary conditions (4) at the surface $y=0$, we obtain a system of four equations. After applying the following inverse of matrix method

$$
\left[\begin{array}{l}
M_{1} \\
M_{2} \\
M_{3} \\
M_{4}
\end{array}\right]=
$$

$$
\left[\begin{array}{cccc}
H_{41} & H_{42} & H_{43} & H_{44} \\
H_{71} & H_{72} & H_{73} & H_{74} \\
v_{31} & v_{32} & v_{33} & v_{34} \\
\left(k_{1}+s_{1}\right) H_{31} & \left(k_{2}+s_{1}\right) H_{32} & \left(k_{3}+s_{1}\right) H_{33} & \left(k_{4}+s_{1}\right) H_{34}
\end{array}\right]^{-1}\left[\begin{array}{c}
-p_{1}^{*} \\
0 \\
p_{2}^{*} \\
0
\end{array}\right]
$$

we obtain the values of coefficients $M_{n}(n=1,2,3,4)$, where

$$
s_{1}=\frac{s C_{T} t^{*}}{D_{E}} .
$$




\section{$5 \quad$ Particular cases}

1. The expressions for the displacement components, force stresses, carrier density and temperature distribution in a rotating generalized semiconducting medium can be obtained from the above equations by taking $a^{*}=0\left(a^{*}=0\right.$ indicates one type temperature).

2. Neglecting the angular velocity (i.e., $\Omega=0$ ) in the above equations, one can obtain the displacement components, carrier density, stress components, conductive temperature and thermodynamic temperature distribution in a non-rotating generalized semiconducting medium with two-temperature.

After substituting $\Omega=0$ in Eq. (5), and use Eqs. (2), (8), and (18), it can be reached that

$$
\begin{gathered}
{\left[D^{2}-\left(a^{2}+\omega^{2}\right)\right] \Phi^{*}+\left(a_{0} D^{2}-b_{3}\right) \theta^{*}-N^{*}=0,} \\
\left(D^{2}-m^{2}\right) \Psi^{*}=0, \\
-b_{6}\left(D^{2}-a^{2}\right) \Phi^{*}+\left(b_{7} D^{2}-b_{8}\right) \theta^{*}+\varepsilon_{2} N^{*}=0, \\
\left(b_{9} D^{2}-b_{10}\right) \theta^{*}-\left(D^{2}-b_{11}\right) N^{*}=0 .
\end{gathered}
$$

Eliminating $\Phi^{*}(y), \theta^{*}(y)$, and $\mathrm{N}^{*}(y)$ in Eqs. (38), (40), and (41), the following sixth order ordinary differential equations for $\Phi^{*}(y), \theta^{*}(y)$, and $N^{*}(y)$ can be obtained

$$
\left(D^{6}-A_{1} D^{4}+B_{1} D^{2}-E_{1}\right)\left[\Phi^{*}(y), \theta^{*}(y), N^{*}(y)\right]=0 .
$$

Equation (42) can be factored as

$$
\left(D^{2}-k_{1}^{2}\right)\left(D^{2}-k_{2}^{2}\right)\left(D^{2}-k_{3}^{2}\right)\left[\Phi^{*}(y), \theta^{*}(y), N^{*}(y)\right]=0,
$$

where $k_{n}^{2}(n=1,2,3)$ are the roots of the characteristic equation of Eq. (43), $m^{2}=a^{2}+\beta^{2} \omega^{2}$,

$A_{1}=\frac{1}{\left(a_{0} b_{6}+b_{7}\right)}\left[\left(a_{0} a^{2}+a_{0} b_{11}+b_{3}\right) b_{6}+\left(a^{2}+\omega^{2}+b_{11}\right) b_{7}+b_{8}+\left(b_{6}-\varepsilon_{2}\right) b_{9}\right]$,

$B_{1}=\frac{1}{\left(a_{0} b_{6}+b_{7}\right)}\left[b_{6}\left(a^{2} a_{0} b_{11}+a^{2} b_{3}+a^{2} b_{9}+b_{10}+b_{3} b_{11}\right)+b_{8} b_{11}-\varepsilon_{2} b_{10}\right.$ $\left.+\left(a^{2}+\omega^{2}\right)\left(b_{8}+b_{7} b_{11}-\varepsilon_{2} b_{9}\right)\right]$,

$E_{1}=\frac{1}{\left(a_{0} b_{6}+b_{7}\right)}\left[\left(a^{2}+\omega^{2}\right)\left(b_{8} b_{11}-\varepsilon_{2} b_{10}\right)+a^{2} b_{6}\left(b_{3} b_{11}+b_{10}\right)\right]$. 
The solution of Eqs. (43) and (39), take the form

$$
\begin{gathered}
\Phi^{*}(y)=\sum_{n=1}^{3} G_{n} e^{-k_{n} y}, \\
\theta^{*}(y)=\sum_{n=1}^{3} R_{1 n} G_{n} e^{-k_{n} y}, \\
\mathrm{~N}^{*}(y)=\sum_{n=1}^{3} R_{2 n} G_{n} e^{-k_{n} y}, \\
\Psi^{*}(y)=G_{4} e^{-m y},
\end{gathered}
$$

where $G_{n}(n=1,2,3,4)$ are some coefficients,

$R_{1 \mathrm{n}}=\frac{\left(k_{n}^{2}-b_{11}\right)\left[k_{n}^{2}-\left(a^{2}+\omega^{2}\right)\right]}{\left[\left(b_{9} k_{n}^{2}-b_{10}\right)-\left(k_{n}^{2}-b_{11}\right)\left(a_{0} k_{n}^{2}-b_{3}\right)\right]}$, and $R_{2 \mathrm{n}}=\frac{\left(b_{9} k_{n}^{2}-b_{10}\right) R_{1 n}}{\left(k_{n}^{2}-b_{11}\right)}$.

Using Eqs. (8), (13)-(18), and (44)-(47), the expressions for the displacement components, the thermodynamic temperature and the stress components distribution in a non-rotating generalized semiconducting medium with two temperature can be written as follows:

$$
\begin{gathered}
u^{*}(y)=\sum_{n=1}^{3} i a G_{n} e^{-k_{n} y}-m G_{4} e^{-m y}, \\
v^{*}(y)=-\sum_{n=1}^{3} k_{n} G_{n} e^{-k_{n} y}-i a G_{4} e^{-m y}, \\
T^{*}(y)=\sum_{n=1}^{3} R_{3 n} G_{n} e^{-k_{n} y}, \\
\sigma_{x x}^{*}=\sum_{n=1}^{3} R_{4 n} G_{n} e^{-k_{n} y}-2 i a m G_{4} e^{-m y}, \\
\sigma_{y y}^{*}=\sum_{n=1}^{3} R_{5 n} G_{n} e^{-k_{n} y}+2 i a m G_{4} e^{-m y}, \\
\sigma_{z z}^{*}=\sum_{n=1}^{3} R_{6 n} G_{n} e^{-k_{n} y},
\end{gathered}
$$




$$
\sigma_{x y}^{*}=-\sum_{n=1}^{3} R_{7 n} G_{n} e^{-k_{n} y}+\left(a^{2}+m^{2}\right) G_{4} e^{-m y}, \quad \sigma_{x z}^{*}=\sigma_{y z}^{*}=0,
$$

where $R_{3 n}=\left[1-a_{0}\left(k_{n}^{2}-a^{2}\right)\right] R_{1 n}, \quad R_{4 n}=\left(\beta^{2}-2\right) k_{n}^{2}-\beta^{2}\left(a^{2}+R_{2 n}+R_{3 n}\right)$, $R_{5 n}=\beta^{2}\left(k_{n}^{2}-R_{2 n}-R_{3 n}\right)-a^{2}\left(\beta^{2}-2\right), \quad R_{6 n}=\left(\beta^{2}-2\right)\left(k_{n}^{2}-a^{2}\right)-\beta^{2}\left(R_{2 n}+\right.$ $\left.R_{3 n}\right), \quad R_{7 n}=2 i a k_{n}$.

Applying the boundary conditions (4) at the surface, $y=0$, a system of four equations is obtained. After solving this system, the coefficients $G_{n}$ $(n=1,2,3,4)$ can be defined as follows:

$$
\begin{gathered}
{\left[\begin{array}{c}
G_{1} \\
G_{2} \\
G_{3} \\
G_{4}
\end{array}\right]=} \\
{\left[\begin{array}{cccc}
R_{41} & R_{42} & R_{43} & -2 i a m \\
R_{71} & R_{72} & R_{73} & -\left(a^{2}+m^{2}\right) \\
R_{31} & R_{32} & R_{33} & 0 \\
\left(k_{1}+s_{1}\right) R_{21} & \left(k_{2}+s_{1}\right) R_{22} & \left(k_{3}+s_{1}\right) R_{23} & 0
\end{array}\right]^{-1}\left[\begin{array}{c}
-p_{1}^{*} \\
0 \\
p_{2}^{*} \\
0
\end{array}\right] .}
\end{gathered}
$$

\section{$6 \quad$ Numerical results}

Silicon, $\mathrm{Si}$, is chosen as the material for numerical simulations. The parameters for silicon are taken as (Song et al. $[11,13]$ ):

$\lambda=3.64 \times 10^{10} \mathrm{~N} \mathrm{~m}^{-2}, \mu=5.46 \times 10^{10} \mathrm{~kg} \mathrm{~m}^{-1} \mathrm{~s}^{-2}, K=150 \mathrm{~W} \mathrm{~m}^{-1} \mathrm{~K}^{-1}$,

$\alpha_{t}=3 \times 10^{-6} \mathrm{~K}^{-1}, \rho=2.33 \times 10^{3} \mathrm{~kg} \mathrm{~m}^{-3}, C_{E}=695 \mathrm{~J} \mathrm{~kg}^{-1} \mathrm{~K}^{-1}$,

$T_{0}=300 \mathrm{~K}, \Omega=0.4 \mathrm{~s}^{-1}, a^{*}=0.4, a=0.5, p_{1}^{*}=p_{2}^{*}=0.01, \tau_{0}=0.01$,

$t=0.02, \quad \varepsilon_{3}=-450, \quad x=0.5, \quad \omega=\omega_{R e}+i \omega_{I m}, \quad \omega=\omega_{R e}=0.6$,

$\omega_{I m}=0, \quad d_{n}=-9 \times 10^{-31} \mathrm{~m}^{3}, \quad D_{E}=2.5 \times 10^{-3} \mathrm{~m}^{2} \mathrm{~s}^{-1}$,

$E_{g}=1.12 e v, \quad \tau=5 \times 10^{-5} \mathrm{~s}, \quad s=2 \mathrm{~m} \mathrm{~s}^{-1}$.

The thermophysical data, outlined above, were used for the determination of the distribution of the real part of displacement components $u, v$, thermodynamic temperature $T$, conductive temperature $\theta$, carrier density $N$, stress components $\sigma_{x x}, \sigma_{y y}, \sigma_{z z}$, and $\sigma_{x y}$ in the presence and absence of the rotation as well as the two temperature parameter. Here, all variables are taken in the non-dimensional form. The results are shown in Figs. 2-10. 
In these figures, the solid lines represent the solution for $\Omega=0.4, a^{*}=0.4$, the dashed ones represent the solution derived for $\Omega=0, a^{*}=0.4$, and the dot-dashed lines represent the solution for $\Omega=0.4, a^{*}=0$. Due to the boundary conditions, the stress components $\sigma_{x x}$ and $\sigma_{x y}$ always start from negative values and zero, respectively, and terminate at a zero value.

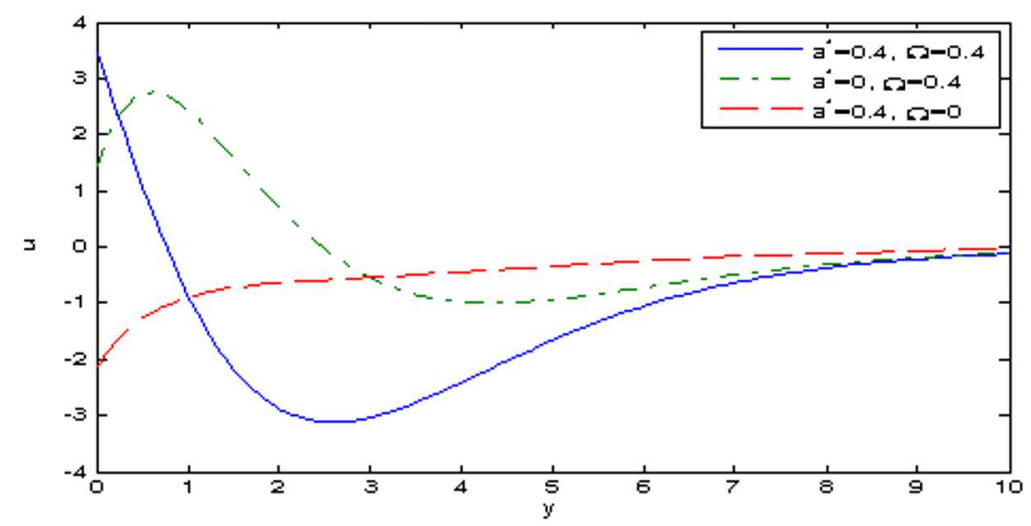

Figure 2: Distribution of horizontal displacement $u$.

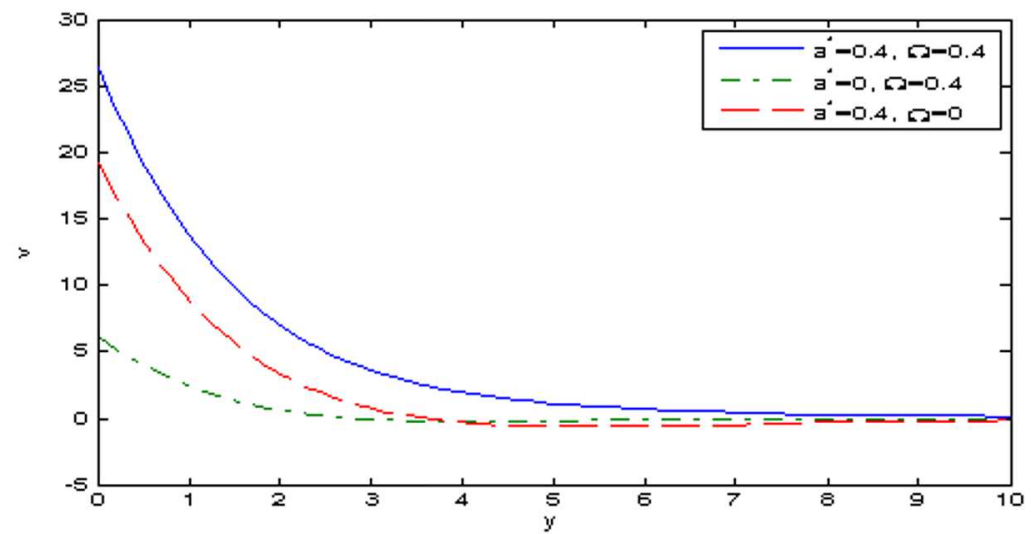

Figure 3: Distribution of vertical displacement $v$.

Figure 2 describes the variation of the horizontal displacement $u$ against the distance $y$. It is clear from Fig. 2 that the values of $u$ decrease in the range $0 \leq y \leq 2.5$, then increase and go to zero in the range $2.5 \leq$ 


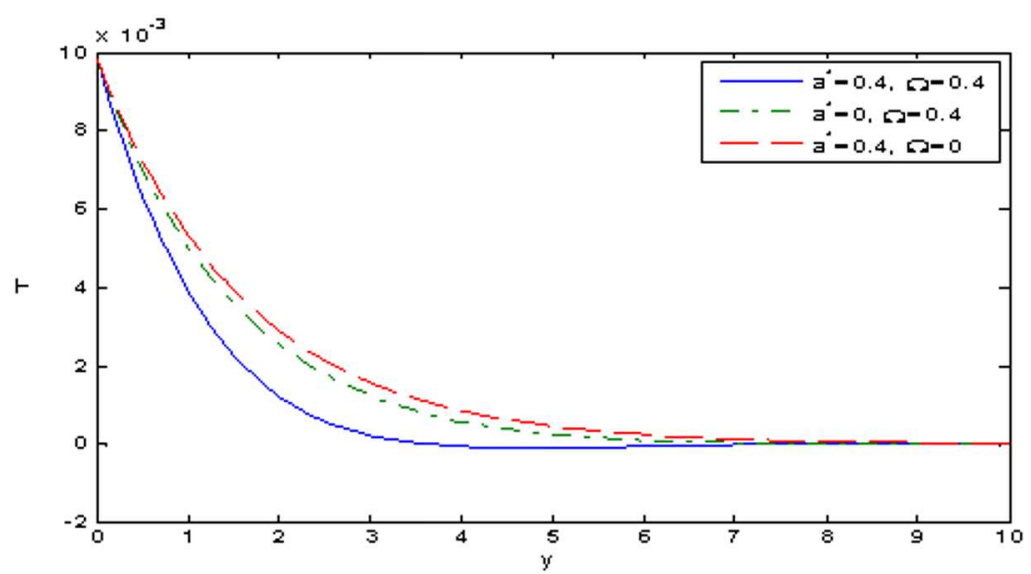

Figure 4: Distribution of the thermodynamic temperature $T$.

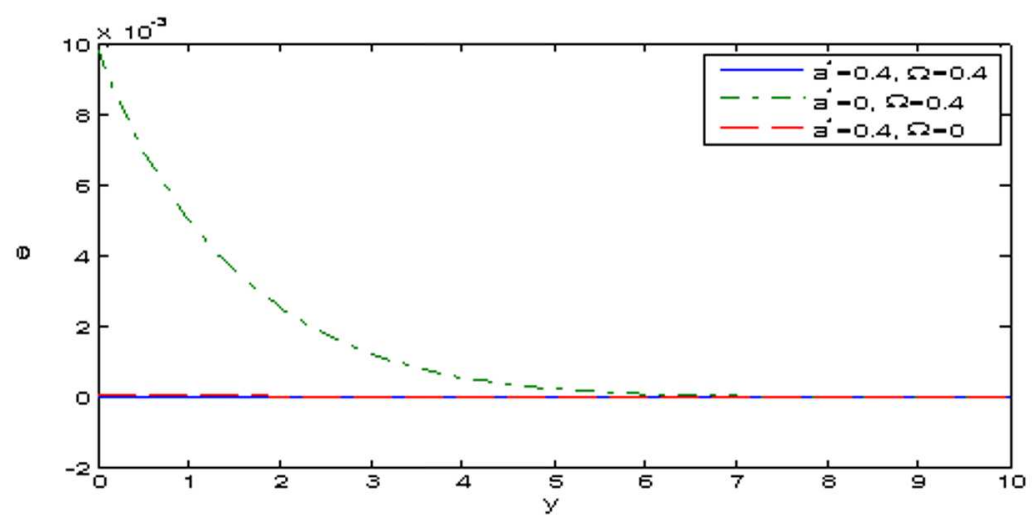

Figure 5: Distribution of the conductive temperature $\theta$.

$y \leq 10$, for $\Omega=0.4, a^{*}=0.4$, while, it increases in the range $0 \leq y \leq$ 0.9 then decrease in the range $0.9 \leq y \leq 5$ and finally increase and go to zero for $\Omega=0.4, a^{*}=0$, while $u$ is an increasing function in the range $0 \leq y \leq 10$ for $\Omega=0, a^{*}=0.4$. Figure 3 shows the variation of the vertical displacement $v$. In this figure, a significant difference in the vertical displacement $v$ is noticed for different values of the two temperature parameter $a^{*}$ as well as rotation. It shows that the magnitude of $v$ for $a^{*}=0.4$ is higher than that of $a^{*}=0$. It is also observed from this figure that the rotation acts to increase the magnitude of the real part of $v$. 


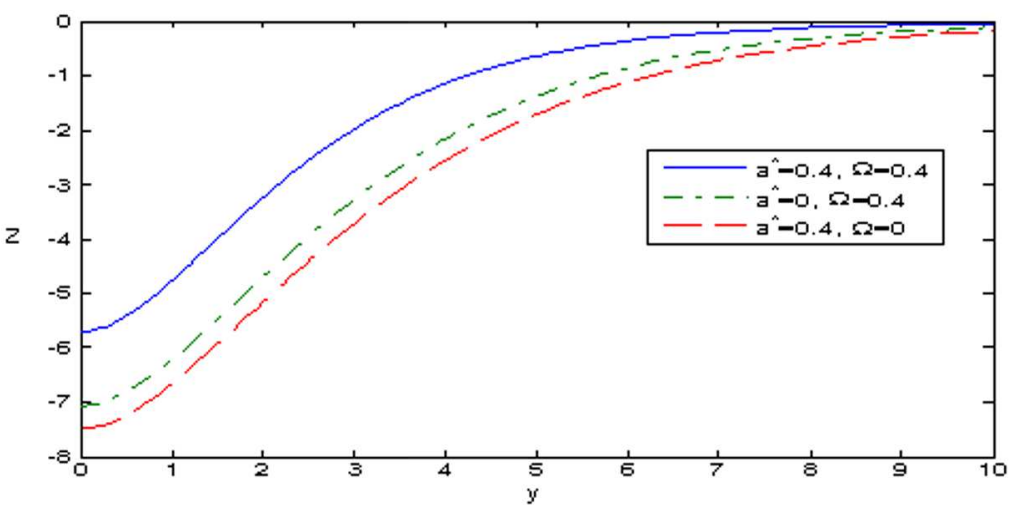

Figure 6: Distribution of the carrier density $N$.

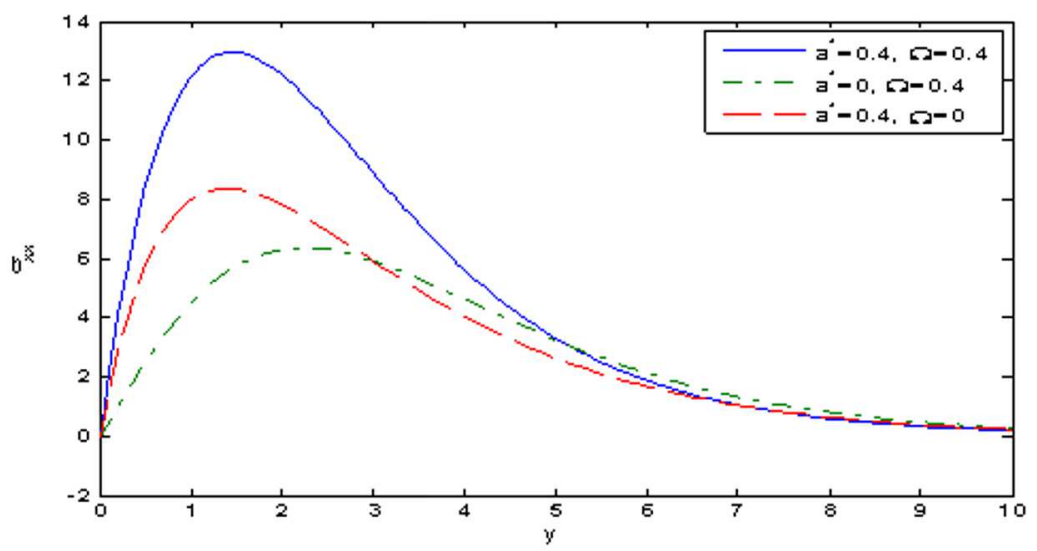

Figure 7: Distribution of stress component $\sigma_{x x}$.

Figure 4 is plotted to show the variation of the thermodynamic temperature $T$. It is observed that the thermodynamic temperature, $T$, decreases in the range $0 \leq y \leq 8$ and finally goes to zero for the different cases. It is also clear that the parameter $a^{*}$ of two-temperature and the rotation, $\Omega$, act to decrease the values of $T$. It is clear that the values of conductive temperature, $\theta$, as shown in Fig. 5, in the two-type temperature cases are small compared to those for one-type temperature case. It is also noticed that the conductive temperature $\theta$ is inversely proportional to the rotation. Figure 6 clarifies that the values of the carrier density $N$ always begin 


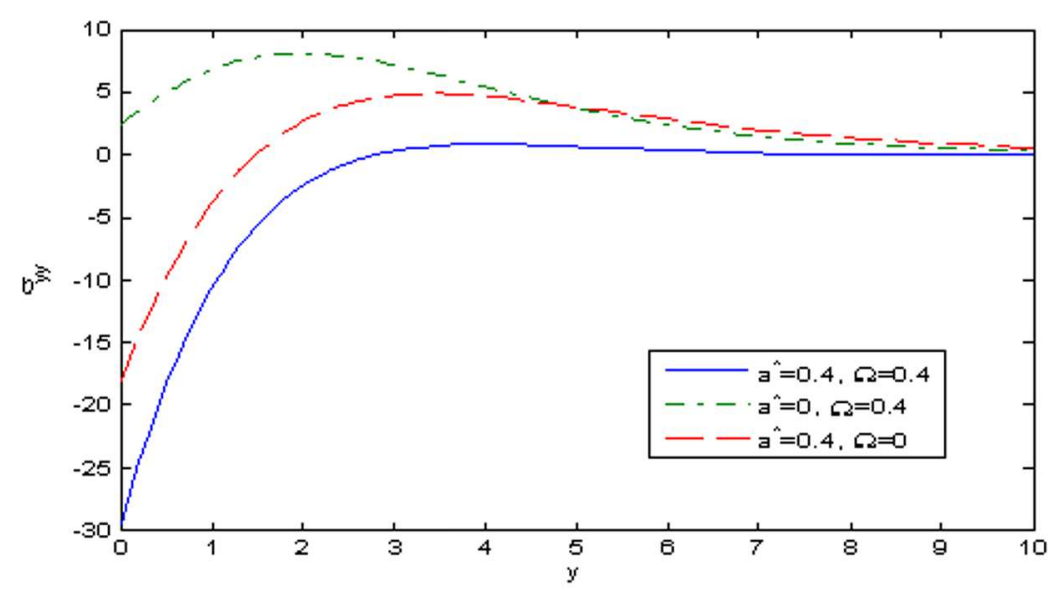

Figure 8: Distribution of stress component $\sigma_{y y}$.

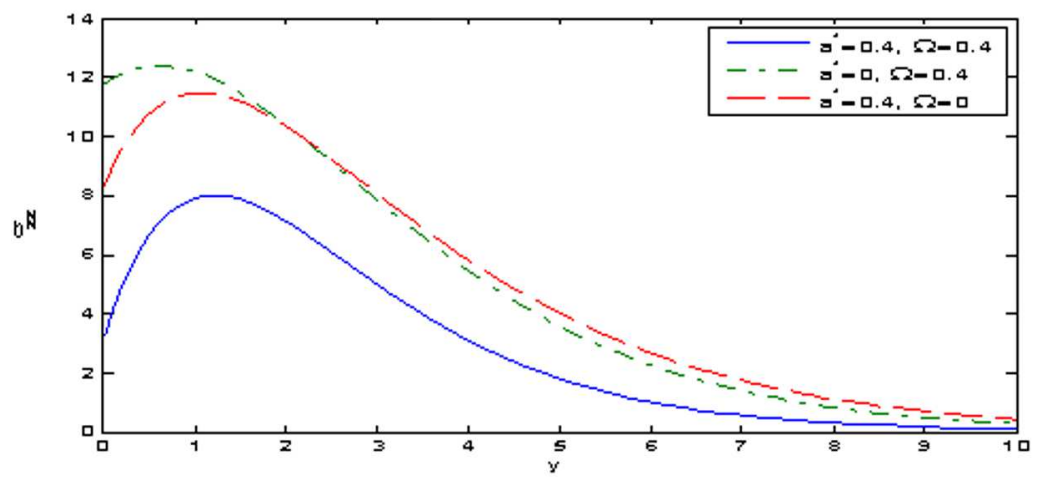

Figure 9: Distribution of stress component $\sigma_{z z}$.

from negative values and increase in the range $0 \leq y \leq 10$, then go to zero for the different cases. It is also seen that the carrier density $N$ is directly proportional to the rotation and the two-temperature parameter. Figure 7 represents the change in the stress component $\sigma_{x x}$ with distance $y$. The values of $\sigma_{x x}$ always start with increasing to a maximum value then decrease and finally go to zero. It is noticed that $\sigma_{x x}$ is strongly affected by the two temperature parameter as well as the rotation. It is directly proportional to both of them. It is clear that the two-temperature parameter $a^{*}$ and the rotation act to decrease the values of $\sigma_{y y}$ as shown 


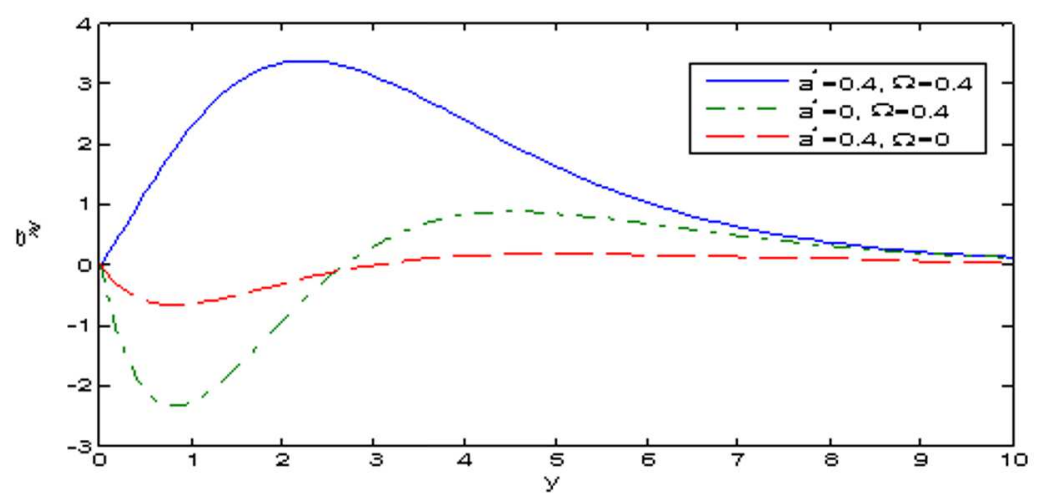

Figure 10: Distribution of stress component $\sigma_{x y}$.

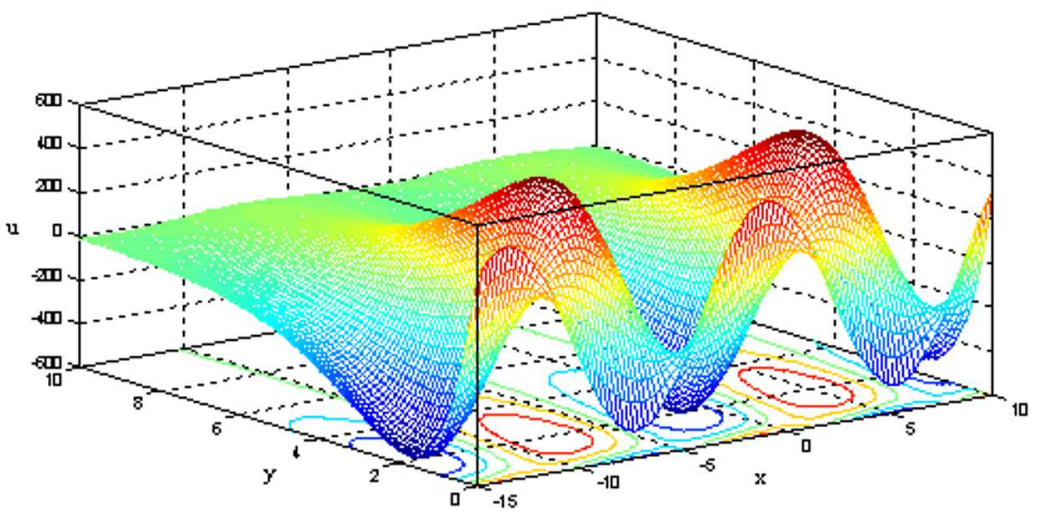

Figure 11: Distribution of the displacement component $u$ versus components of distance at $\Omega=0.4$ and $a^{*}=0.4$.

in Fig. 8. Figure 9 depicts that the distribution of stress component $\sigma_{z z}$ always begins from positive values. For different cases, the values of $\sigma_{z z}$ start with increasing to a maximum value in the range $0 \leq y \leq 1.2$, then decrease in the range $1.2 \leq y \leq 10$ and finally tend to zero. It is clear that the two-temperature parameter $a^{*}$ as well as, the rotation acts to decrease the values of $\sigma_{z z}$. Figure 9 explains that the distribution of the stress component $\sigma_{x y}$ always starts with zero at the origin which agrees with the boundary conditions. It is clear that the two-temperature parameter $a^{*}$ acts to increase the values of $\sigma_{x y}$. It can be also observed from this figure 


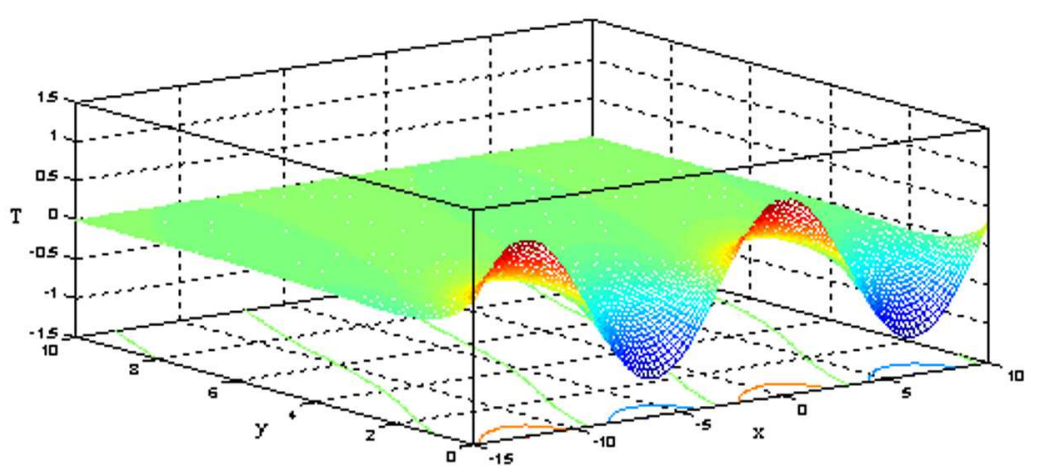

Figure 12: Distribution of the thermodynamic temperature $T$ versus components of distance at $\Omega=0.4$ and $a^{*}=0.4$.

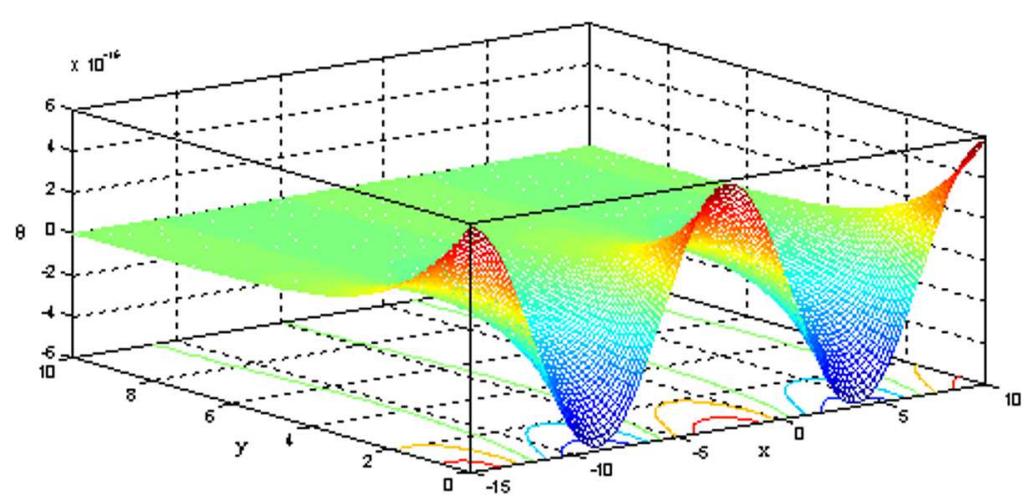

Figure 13: Distribution of the conductive temperature $\theta$ versus components of distance at $\Omega=0.4$ and $a^{*}=0.4$.

that the values of the stress component $\sigma_{x y}$ in the presence of rotation are higher than those in the absence of rotation. Figures 10-15 depict the $3 \mathrm{D}$ curves which represent the relation between the physical quantities and both components of distance in the context of the (L-S) theory for $\Omega=0.4$, and $a^{*}=0.4$, these figures are very important to study the dependence of these physical quantities on the vertical component of distance. The curves obtained are highly depending on the vertical distance and all the physical quantities are moving in the wave propagation. 


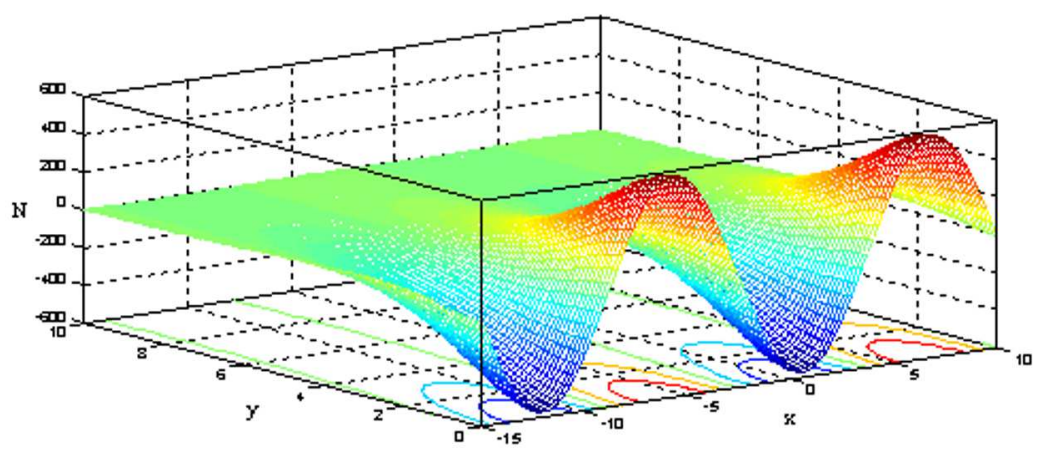

Figure 14: Distribution of carrier density $N$ versus components of distance at $\Omega=0.4$ and $a^{*}=0.4$.

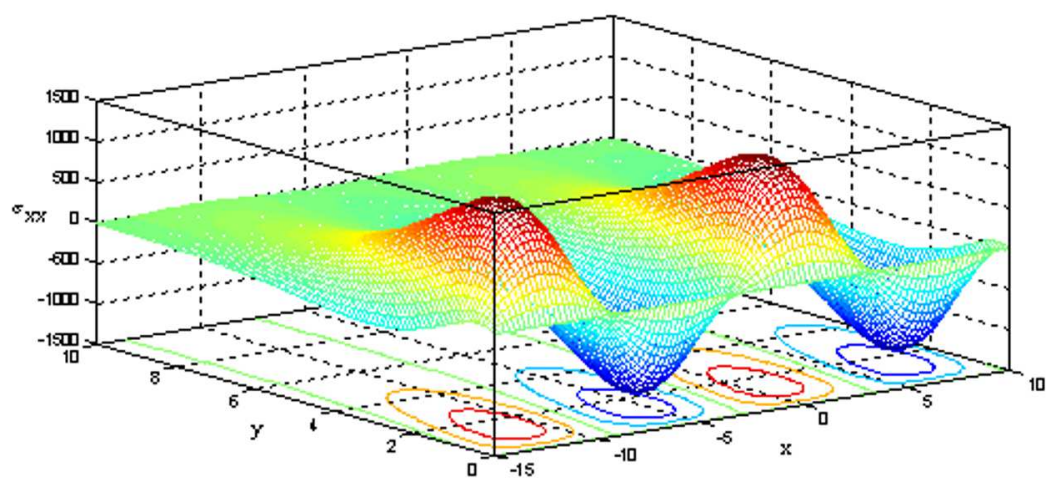

Figure 15: Distribution of stress component $\sigma_{x x}$ versus components of distance at $\Omega=0.4$ and $a^{*}=0.4$.

\section{Conclusion}

According to the results of this work, one can see the effect of rotation as well as of the two temperature parameter on the wave propagation of all fields and how they play a vital role in increasing or decreasing the amplitude of different physical quantities. This work proves the importance of distinguishing between the conductive temperature and the thermodynamic temperature. Also, the figures show that the presence of either the two temperature parameter or the rotation has the same effect on the different physical quantities. This work can serve for the analysis and design 


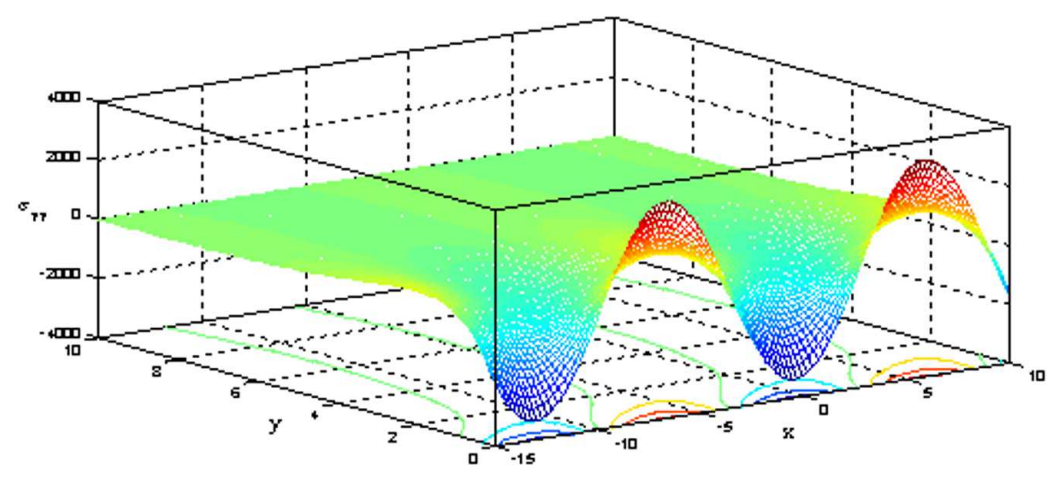

Figure 16: Distribution of stress component $\sigma_{y y}$ versus components of distance at $\Omega=0.4$ and $a^{*}=0.4$.

of the thermal resistance coated materials. There are a lot of applications on diverse field as semiconducting and the reactions during a photothermal process and other fields in physical engineering, electronic devices, transistors, also in physical chemistry and medical physics.

Received 2 November 2016

\section{References}

[1] Biot M.A.: Thermoelasticity and irreversible thermodynamics. J. Appl. Phys. 27(1956), 3, 240-253.

[2] Lord H.W., Shulman Y.A.: Generalized dynamical theory of thermo-elasticity. J. Mech. Phys. Solids. 15(1967), 5, 299-30.

[3] Maxwell J.C.: On the dynamical theory of gases. J. Philos. Trans. Roy. Soc. London. 157(1867), 49-88.

[4] Cattaneo C.: Sur une forme de l'equation de la chaleur elinant le paradoxe d'une propagation instantance. CR Acad. Sci. 247(1958), 431-432 (in France).

[5] Vernotte P.: Les paradoxes de la theorie continue de l'equation de la chaleur. CR Acad. Sci. 246(1958), 3154-3155 (in Franch).

[6] Dhaliwal R., Sherief H.H.: Generalized thermoelasticity for anisotropic media. Quart. Appl. Math. 33(1980), 1, 1-8.

[7] Othman M.I.A., SAID S.M.: The effect of rotation on two-dimensional problem of a fiber-reinforced thermoelastic with one relaxation time. Int. J. Thermophysics. 33(2012), 1, 160-171. 
[8] Gordon J. P., Leite R.C.C., Moore R.S.: Long-transient effects in lasers with inserted liquid samples. J. Appl. Phys. 36(1965), 1, 3-8.

[9] KReuzer L.B.: Ultralow gas concentration infrared absorption spectroscopy. J. Appl. Phys. 42(1971), 7, 2934-2943.

[10] Todorovic D.M., Nikolic P.M., BoJicic A.I.: Photoacoustic frequency transmission technique: Electronic deformation mechanism in semiconductors. J. Appl. Phys. 85(1999), 7716-7726.

[11] Song Y.Q., Todorovic D.M., Cretin B.: Study on the generalized thermoelastic vibration of the optically excited semiconducting micro-cantilevers. Int. J. Solids Struct. 47(2010), 14-15, 1871-1875.

[12] Todorovic D.M.: Plasma, thermal, and elastic waves in semiconductors. Rev. Sci. Instrum. 74(2003), 1, 582-585.

[13] Song Y.Q., BAI J.T., REN Z.Y.: Study on the reflection of photothermal waves in a semiconducting medium under generalized thermoelastic theory. Acta Mech. 223(2012), 7, 1545-1557.

[14] Schoenberg M., Censor D.: Elastic waves in rotating media. Q. J. Appl. Maths. 31(1973), 1, 115-125.

[15] Chand D., Sharma J.N., Sud S.P.: Transient generalized magneto-thermo-elastic waves in a rotating half space. Int. J. Eng. Sci. 28(1990), 547-556.

[16] Destrade M.: Surface waves in rotating rhombic crystal. Proc. Royal Soc. A 460(2004), 653-665.

[17] Oтhмan M.I.A.: Effect of rotation in the case of 2-D problems of the generalized thermoelasticity with thermal relaxation. Mech. Mech. Eng. 9(2005), 2, 115-130.

[18] Othman M.I.A., Singh B.: The effect of rotation on generalized micropolar thermoelasticity for a half-space under five theories. Int. J. Solids Struct. 44(2007), $2748-2762$.

[19] Othman M.I.A, Hasona W.M., Eraki E.E.M.: Effect of magnetic field on generalized thermoelastic rotating medium with two temperature under five theories. J. Comput, Theor. Nanos. 12(2015), 8, 1677-1686.

[20] Hayat T., Mumtaz S., Ellahi R.: MHD unsteady flows due to non- coaxial rotations of a disk and a fluid at infinity. Acta Mech. Sinica. 19(2003), 3, 235-240.

[21] Hayat T., Ellahi R., Asghar S., Siddiqui A.M.: Flow induced by non-coaxial rotation of a porous disk executing non-torsional oscillating and second grade fluid rotating at infinity. Appl. Math. Model. 28(2004), 6, 591-605.

[22] Hayat T., Ellahi R., Asghar S.: Unsteady periodic flows of a magnetohydrodynamic fluid due to non- coaxial rotations of a porous disk and fluid at infinity. Math. Comput. Model. 40(2004), No. 1-2, 173-179.

[23] Hayat T., Ellahi R., Asghar S.: Unsteady magnetohydrodynamic non-Newtonian flow due to non-coaxial rotations of a disk and a fluid at infinity. Chem. Eng. Commun. 194(2007), 1, 37-49.

[24] Ellahi R., Asgahr S.: Couette flow of a Burgers' fluid with rotation. Int. J. Fluid Mech. Res. 34(2007), 548-561. 
[25] Hayat T., Ellahi R., Asghar S.: Hall effects on unsteady flow due to noncoaxially rotating disk and a fluid at infinity. Chem. Eng. Commun. 195(2008), 8, 958-976.

[26] Chen P.J., Gurtin M.E.: On a theory of heat conduction involving two temperatures. ZAMP 19(1968), 4, 614-627.

[27] Chen P. J., Gurtin M.E., Williams W.O.: A note on non-simple heat conduction. ZAMP 19(1968), 6, 969-970.

[28] Chen P.J., Gurtin M.E., Williams W.O.: On the thermodynamics of non-simple elastic materials with two temperatures. ZAMP 20(1969), 1, 107-112.

[29] BOLEY B. A., SnplaceTOLINS SnI. S.: Transient coupled thermoelastic boundary value problems in the half-space. J. App. Mech. 29(1962), 4, 637-646.

[30] Warren W.E., Chen P.J.: Wave propagation in the two-temperature theory of thermoelasticity. Acta Mech. 16(1973), 1-2, 21-33.

[31] Youssef H.M.: Theory of two-temperature generalized thermoelasticity. IMAJ. Appl. Math. 71(2006), 3, 383-390.

[32] Youssef H.M.: Two-dimensional problem of two-temperature generalized thermoelastic half-space subjected to Ramp-type heating. J. Comp. Math. Model. 19(2008), 201-216.

[33] AbBas I.A., Youssef H.M.: Two-temperature generalized thermoelasticity under ramp-type heating by finite element method. Meccanica 48(2013), 2, 331-339.

[34] BiJARniA R., Singh B.: propagation of plane waves in a rotating transversely isotropic two temperature generalized thermoelastic solid half-space with voids. Int. J. of Appl. Mech. Eng. 21(2016), 1, 285-301.

[35] Kaviany M.: Heat Transfer Physics. Cambridge Univ. Press, Cambridge 2008. 